\title{
AN INVERSE SCATTERING PROBLEM FOR SHORT-RANGE SYSTEMS IN A TIME-PERIODIC ELECTRIC FIELD.
}

\author{
François Nicoleau
}

\begin{abstract}
We consider a time-dependent Hamiltonian $H(t)=\frac{1}{2} p^{2}-E(t) \cdot x+$ $V(t, x)$ on $L^{2}\left(\mathbb{R}^{n}\right)$, where the external electric field $E(t)$ and the short-range electric potential $V(t, x)$ are time-periodic with the same period. It is well-known that the short-range notion depends on the mean value $E_{0}$ of the external electric field. When $E_{0}=0$, we show that the high energy limit of the scattering operators determines uniquely $V(t, x)$. When $E_{0} \neq 0$, the same result holds in dimension $n \geq 3$ for generic short-range potentials. In dimension $n=2$, one has to assume a stronger decay on the electric potential.
\end{abstract}

\section{Introduction}

In this note, we study an inverse scattering problem for a two-body short-range system in the presence of an external time-periodic electric field $E(t)$ and a timeperiodic short-range potential $V(t, x)$ (with the same period $T$ ). For the sake of simplicity, we assume that the period $T=1$.

The corresponding Hamiltonian is given on $L^{2}\left(\mathbb{R}^{n}\right)$ by :

$$
H(t)=\frac{1}{2} p^{2}-E(t) \cdot x+V(t, x),
$$

where $p=-i \partial_{x}$. When $E(t)=0$, the Hamiltonian $H(t)$ describes the dynamics of the hydrogen atom placed in a linearly polarized monochromatic electric field, or a light particle in the restricted three-body problem in which two other heavy particles are set on prescribed periodic orbits. When $E(t)=\cos (2 \pi t) E$ with $E \in \mathbb{R}^{n}$, the Hamiltonian describes the well-known AC-Stark effect in the $E$ direction [7].

In this paper, we assume that the external electric field $E(t)$ satisfies :

$$
t \rightarrow E(t) \in L_{l o c}^{1}\left(\mathbb{R} ; \mathbb{R}^{n}\right) \quad, \quad E(t+1)=E(t) \text { a.e } .
$$

Moreover, we assume that the potential $V \in C^{\infty}\left(\mathbb{R} \times \mathbb{R}^{n}\right)$, is time-periodic with period 1, and satisfies the following estimations :

$$
\forall \alpha \in \mathbb{N}^{n}, \forall k \in \mathbb{N},\left|\partial_{t}^{k} \partial_{x}^{\alpha} V(t, x)\right| \leq C_{k, \alpha}<x>^{-\delta-|\alpha|} \text {, with } \delta>0,
$$

where $\langle x\rangle=\left(1+x^{2}\right)^{\frac{1}{2}}$. Actually, we can accommodate more singular potentials (see [10], [11], [12] for example) and we need $\left(A_{2}\right)$ for only $k, \alpha$ with finite order . It is well-known that under assumptions $\left(A_{1}\right)-\left(A_{2}\right), H(t)$ is essentially

Received by the editors June 28, 2005. 
self-adjoint on $\mathcal{S}\left(\mathbb{R}^{n}\right)$ the Schwartz space, [16]. We denote $H(t)$ the self-adjoint realization with domain $D(H(t))$.

Now, let us recall some well-known results in scattering theory for time-periodic electric fields. We denote $H_{0}(t)$ the free Hamiltonian :

$$
H_{0}(t)=\frac{1}{2} p^{2}-E(t) \cdot x,
$$

and let $U_{0}(t, s)$, (resp. $\left.U(t, s)\right)$ be the unitary propagator associated with $H_{0}(t)$, (resp. $H(t)$ ) (see section 2 for details).

For short-range potentials, the wave operators are defined for $s \in \mathbb{R}$ and $\Phi \in$ $L^{2}\left(\mathbb{R}^{n}\right)$ by :

$$
W^{ \pm}(s) \Phi=\lim _{t \rightarrow \pm \infty} U(s, t) U_{0}(t, s) \Phi .
$$

We emphasize that the short-range condition depends on the value of the mean of the external electric field :

$$
E_{0}=\int_{0}^{1} E(t) d t
$$

- The case $E_{0}=0$

By virtue of the Avron-Herbst formula (see section 2), this case falls under the category of two-body systems with time-periodic potentials and this case was studied by Kitada and Yajima ([10], [11]), Yokoyama [22].

We recall that for a unitary or self-adjoint operator $U, \mathcal{H}_{c}(U), \mathcal{H}_{a c}(U), \mathcal{H}_{s c}(U)$ and $\mathcal{H}_{p}(U)$ are, respectively, continuous, absolutely continuous, singular continuous and point spectral subspace of $U$.

We have the following result ([10], [11], [21]) :

\section{Theorem 1.}

Assume that hypotheses $\left(A_{1}\right),\left(A_{2}\right)$ are satisfied with $\delta>1$ and with $E_{0}=0$. Then : (i) the wave operators $W^{ \pm}(s)$ exist for all $s \in \mathbb{R}$.

(ii) $W^{ \pm}(s+1)=W^{ \pm}(s)$ and $U(s+1, s) W^{ \pm}(s)=W^{ \pm}(s) U_{0}(s+1, s)$.

(iii) $\operatorname{Ran}\left(W^{ \pm}(s)\right)=\mathcal{H}_{a c}(U(s+1, s))$ and $\mathcal{H}_{s c}(U(s+1, s))=\emptyset$.

(iv) the purely point spectrum $\sigma_{p}(U(s+1, s))$ is discrete outside $\{1\}$.

\section{Comments}

1 - The unitary operators $U(s+1, s)$ are called the Floquet operators and they are mutually equivalent. The Floquet operators play a central role in the analysis of time periodic systems.

The eigenvalues of these operators are called Floquet multipliers. In [5], Galtbayar, Jensen and Yajima improve assertion $(i v)$ : for $n=3$ and $\delta>2$, $\mathcal{H}_{p}(U(s+1, s))$ is finite dimensional. 
2 - For general $\delta>0, W^{ \pm}(s)$ do not exist and we have to define other wave operators. In ([10], [11]), Kitada and Yajima have constructed modified wave operators $W_{H J}^{ \pm}$by solving an Hamilton-Jacobi equation.

- The case $E_{0} \neq 0$

This case was studied by Moller [12] : using the Avron-Herbst formula, it suffices to examine Hamiltonians with a constant external electric field, (Stark Hamiltonians); the spectral and the scattering theory for Stark Hamiltonians are well established [2]. In particular, a Stark Hamiltonian with a potential $V$ satisfying $\left(A_{2}\right)$ has no eigenvalues [2]. The following theorem, due to Moller [12], is a time-periodic version of these results.

\section{Theorem 2.}

Assume that hypotheses $\left(A_{1}\right),\left(A_{2}\right)$ are satisfied with $\delta>\frac{1}{2}$ and with $E_{0} \neq 0$.

Then: (i) the Floquet operators have purely absolutely continuous spectrum.

(ii) the wave operators $W^{ \pm}(s)$ exist for all $s \in \mathbb{R}$ and are unitary.

(iii) $W^{ \pm}(s+1)=W^{ \pm}(s)$ and $U(s+1, s) W^{ \pm}(s)=W^{ \pm}(s) U_{0}(s+1, s)$.

\section{The inverse scattering problem}

For $s \in \mathbb{R}$, we define the scattering operators $S(s)=W^{+*}(s) W^{-}(s)$. It is clear that the scattering operators $S(s)$ are periodic with period 1.

The inverse scattering problem consists to reconstruct the perturbation $V(s, x)$ from the scattering operators $S(s), s \in[0,1]$.

In this paper, we prove the following result :

\section{Theorem 3.}

Assume that $E(t)$ satisfies $\left(A_{1}\right)$ and let $V_{j}, j=1,2$ be potentials satisfying $\left(A_{2}\right)$. We assume that $\delta>1$ (if $E_{0}=0$ ), $\delta>\frac{1}{2}$ (if $E_{0} \neq 0$ and $n \geq 3$ ), $\delta>\frac{3}{4}$ (if $E_{0} \neq 0$ and $n=2$ ).

Let $S_{j}(s)$ be the corresponding scattering operators.

Then :

$$
\forall s \in[0,1], S_{1}(s)=S_{2}(s) \Longrightarrow V_{1}=V_{2}
$$

We prove Theorem 3 by studying the high energy limit of $[S(s), p]$, (Enss-Weder's approach [4]). We need $n \geq 3$ in the case $E_{0} \neq 0$ in order to use the inversion of the Radon transform [6] on the orthogonal hyperplane to $E_{0}$. See also [15] for a similar problem with a Stark Hamiltonian.

We can also remark that if we know the free propagator $U_{0}(t, s), s, t \in \mathbb{R}$, then by virtue of the following relation :

$$
S(t)=U_{0}(t, s) S(s) U_{0}(s, t),
$$


the potential $V(t, x)$ is uniquely reconstructed from the scattering operator $S(s)$ at only one initial time.

In [21], Yajima proves uniqueness for the case of time-periodic potential with the condition $\delta>\frac{n}{2}+1$ and with $E(t)=0$ by studying the scattering matrices in a high energy regime.

In [20], for a time-periodic potential that decays exponentially at infinity, Weder proves uniqueness at a fixed quasi-energy.

Note also that inverse scattering for long-range time-dependent potentials without external electric fields was studied by Weder [18] with the Enss-Weder timedependent method, and by Ito for time-dependent electromagnetic potentials for Dirac equations [8].

\section{Proof of Theorem 3}

2.1. The Avron-Herbst formula. First, let us recall some basic definitions for time-dependent Hamiltonians. Let $\{H(t)\}_{t \in \mathbb{R}}$ be a family of selfadjoint operators on $L^{2}\left(\mathbb{R}^{n}\right)$ such that $\mathcal{S}\left(\mathbb{R}^{n}\right) \subset D(H(t))$ for all $t \in \mathbb{R}$.

\section{Definition.}

We call propagator a family of unitary operators on $L^{2}\left(\mathbb{R}^{n}\right), U(t, s), t, s \in \mathbb{R}$ such that:

1 - $U(t, s)$ is a strongly continuous fonction of $(t, s) \in \mathbb{R}^{2}$.

$2-U(t, s) U(s, r)=U(t, r)$ for all $t, s, r \in \mathbb{R}$.

$3-U(t, s)\left(\mathcal{S}\left(\mathbb{R}^{n}\right)\right) \subset \mathcal{S}\left(\mathbb{R}^{n}\right)$ for all $t, s \in \mathbb{R}$.

4 - If $\Phi \in \mathcal{S}\left(\mathbb{R}^{n}\right), U(t, s) \Phi$ is continuously differentiable in $t, s$ and satisfies :

$$
i \frac{\partial}{\partial t} U(t, s) \Phi=H(t) U(t, s) \Phi, \quad i \frac{\partial}{\partial s} U(t, s) \Phi=-U(t, s) H(s) \Phi .
$$

To prove the existence and the uniqueness of the propagator for our Hamiltonians $H(t)$, we use a generalization of the Avron-Herbst formula close to the one given in [3].

In [12], the author gives, for $E_{0} \neq 0$, a different formula which has the advantage to be time-periodic. To study our inverse scattering problem, we use here a different one, which is defined for all $E_{0}$. We emphasize that with our choice, $c(t)$ (see below for the definition of $c(t)$ ) is also periodic with period 1; in particular $c(t)=O(1)$.

The basic idea is to generalize the well-known Avron-Herbst formula for a Stark Hamiltonian with a constant electric field $E_{0}$, [2]; if we consider the Hamiltonian $B_{0}$ on $L^{2}\left(\mathbb{R}^{n}\right)$,

$$
B_{0}=\frac{1}{2} p^{2}-E_{0} \cdot x
$$


we have the following formula :

$$
e^{-i t B_{0}}=e^{-i \frac{E_{0}^{2}}{6} t^{3}} e^{i t E_{0} \cdot x} e^{-i \frac{t^{2}}{2} E_{0} \cdot p} e^{-i t \frac{p^{2}}{2}} .
$$

In the next definition, we give a similar formula for time-dependent electric fields.

\section{Definition}

We consider the family of unitary operators $T(t)$, for $t \in \mathbb{R}$ :

$$
T(t)=e^{-i a(t)} e^{-i b(t) \cdot x} e^{-i c(t) \cdot p},
$$

where :

$$
\begin{gathered}
b(t)=-\int_{0}^{t}\left(E(s)-E_{0}\right) d s-\int_{0}^{1} \int_{0}^{t}\left(E(s)-E_{0}\right) d s d t \\
c(t)=-\int_{0}^{t} b(s) d s . \\
a(t)=\int_{0}^{t}\left(\frac{1}{2} b^{2}(s)-E_{0} \cdot c(s)\right) d s .
\end{gathered}
$$

\section{Lemma 4.}

The family $\left\{H_{0}(t)\right\}_{t \in \mathbb{R}}$ has an unique propagator $U_{0}(t, s)$ defined by :

$$
U_{0}(t, s)=T(t) e^{-i(t-s) B_{0}} T^{*}(s) .
$$

Proof.

We can always assume $s=0$ and we make the following ansatz:

$$
U_{0}(t, 0)=e^{-i a(t)} e^{-i b(t) \cdot x} e^{-i c(t) \cdot p} e^{-i t B_{0}} .
$$

Since on the Schwartz space, $U_{0}(t, 0)$ must satisfy :

$$
i \frac{\partial}{\partial t} U_{0}(t, 0)=H_{0}(t) U_{0}(t, 0)
$$

the functions $a(t), b(t), c(t)$ solve :

$$
\dot{b}(t)=-E(t)+E_{0}, \dot{c}(t)=-b(t), \dot{a}(t)=\frac{1}{2} b^{2}(t)-E_{0} \cdot c(t) .
$$

We refer to [3] for details and [12] for a different formula.

In the same way, in order to define the propagator corresponding to the family $\{H(t)\}$, we consider a Stark Hamiltonian with a time-periodic potential : $B(t)=$ $B_{0}+V_{1}(t, x)$ where

$$
V_{1}(t, x)=e^{i c(t) \cdot p} V(t, x) e^{-i c(t) \cdot p}=V(t, x+c(t)),
$$


(we recall that $c(t)$ a is $C^{1}$-periodic function). Then, $B(t)$ has an unique propagator $R(t, s)$, (see [16] for the case $E_{0}=0$ and [12] for the case $E_{0} \neq 0$ ). It is easy to see that the propagator $U(t, s)$ for the family $\{H(t)\}$ is defined by :

$$
U(t, s)=T(t) R(t, s) T^{*}(s) .
$$

\section{Comments}

Since the Hamiltonians $H_{0}(t)$ and $H(t)$ are time-periodic with period 1, one has for all $t, s \in \mathbb{R}$ :

$$
U_{0}(t+1, s+1)=U_{0}(t, s), U(t+1, s+1)=U(t, s) .
$$

Thus, the wave operators satisfy $W^{ \pm}(s+1)=W^{ \pm}(s)$.

2.2. The high energy limit of the scattering operators. In this section, we study the high energy limit of the scattering operators by using the well-known Enss-Weder's time-dependent method [4]. This method can be used to study Hamiltonians with electric and magnetic potentials on $L^{2}\left(\mathbb{R}^{n}\right)[1]$, the Dirac equation [9], the N-body case [4], the Stark effect ([15], [17]), the AharonovBohm effect [18].

In [13], [14] a stationary approach, based on the same ideas, is proposed to solve scattering inverse problems for Schrödinger operators with magnetic fields or with the Aharonov-Bohm effect.

Before giving the main result of this section, we need some notation.

- $\Phi, \Psi$ are the Fourier transforms of functions in $C_{0}^{\infty}\left(\mathbb{R}^{n}\right)$.

- $\omega \in S^{n-1} \cap \Pi_{E_{0}}$ is fixed, where $\Pi_{E_{0}}$ is the orthogonal hyperplane to $E_{0}$.

- $\Phi_{\lambda, \omega}=e^{i \sqrt{\lambda} x \cdot \omega} \Phi, \Psi_{\lambda, \omega}=e^{i \sqrt{\lambda} x \cdot \omega} \Psi$.

We have the following high energy asymptotics where $<,>$ is the usual scalar product in $L^{2}\left(\mathbb{R}^{n}\right)$ :

\section{Proposition 5.}

Under the assumptions of Theorem 3, we have for all $s \in[0,1]$,

$$
\begin{aligned}
<[S(s), p] \Phi_{\lambda, \omega}, \Psi_{\lambda, \omega}>= & \lambda^{-\frac{1}{2}}<\left(\int_{-\infty}^{+\infty} \partial_{x} V(s, x+t \omega) d t\right) \Phi, \Psi> \\
& +o\left(\lambda^{-\frac{1}{2}}\right) .
\end{aligned}
$$

\section{Comments}

Actually, for the case $n=2, E_{0} \neq 0$ and $\delta>\frac{3}{4}$, Proposition 5 is also valid for $\omega \in S^{n-1}$ satisfying $\left|\omega \cdot E_{0}\right|<\left|E_{0}\right|$, (see ([18], [15]).

Then, Theorem 3 follows from Proposition 5 and the inversion of Radon transform ([6] and [15], Section 2.3). 


\section{Proof of Proposition 5}

For example, let us prove Proposition 5 for the case $E_{0} \neq 0$ and $n \geq 3$, the other cases are similar. More precisely, see [18] for the case $E_{0}=0$, and for the case $n=2, E_{0} \neq 0$, see ([17], Theorem 2.4) and ([15], Theorem 4).

\section{- Step 1}

Since $c(t)$ is periodic, $c(t)=O(1)$. Then, $V_{1}(t, x)$ is a short-range perturbation of $B_{0}$, and we can define the usual wave operators for the pair of Hamiltonians $\left(B(t), B_{0}\right)$ :

$$
\Omega^{ \pm}(s)=\mathrm{s}-\lim _{t \rightarrow \pm \infty} R(s, t) e^{-i(t-s) B_{0}}
$$

Consider also the scattering operators $S_{1}(s)=\Omega^{+*}(s) \Omega^{-}(s)$. By virtue of $(2.6)$ and (2.11), it is clear that :

$$
S(s)=T(s) S_{1}(s) T^{*}(s)
$$

Using the fact that $e^{-i b(s) \cdot x} p e^{i b(s) \cdot x}=p+b(s)$, we have :

$$
[S(s), p]=[S(s), p+b(s)]=T(s)\left[S_{1}(s), p\right] T^{*}(s) .
$$

Thus,

$$
<[S(s), p] \Phi_{\lambda, \omega}, \Psi_{\lambda, \omega}>=<\left[S_{1}(s), p\right] T^{*}(s) \Phi_{\lambda, \omega}, T^{*}(s) \Psi_{\lambda, \omega}>.
$$

On the other hand,

$$
T^{*}(s) \Phi_{\lambda, \omega}=e^{i \sqrt{\lambda} x \cdot \omega} e^{i c(s) \cdot(p+\sqrt{\lambda} \omega)} e^{i b(s) \cdot x} e^{i a(s)} \Phi .
$$

So, we obtain :

$$
<[S(s), p] \Phi_{\lambda, \omega}, \Psi_{\lambda, \omega}>=<\left[S_{1}(s), p\right] f_{\lambda, \omega}, g_{\lambda, \omega}>,
$$

where

$$
f=e^{i c(s) \cdot p} e^{i b(s) \cdot x} \Phi \text { and } g=e^{i c(s) \cdot p} e^{i b(s) \cdot x} \Psi .
$$

Clearly, $f, g$ are the Fourier transforms of functions in $C_{0}^{\infty}\left(\mathbb{R}^{n}\right)$.

\section{- Step 2 : Modified wave operators}

Now, we follow a strategy close to [15] for time-dependent potentials. First, let us define a free-modified dynamic $U_{D}(t, s)$ by :

$$
U_{D}(t, s)=e^{-i(t-s) B_{0}} e^{-i \int_{0}^{t-s} V_{1}\left(u+s, u p^{\prime}+\frac{1}{2} u^{2} E_{0}\right) d u},
$$

where $p^{\prime}$ is the projection of $p$ on the orthogonal hyperplane to $E_{0}$.

We define the modified wave operators :

$$
\Omega_{D}^{ \pm}(s)=\mathrm{s}-\lim _{t \rightarrow \pm \infty} R(s, t) U_{D}(t, s) .
$$

It is clear that :

$$
\Omega_{D}^{ \pm}(s)=\Omega^{ \pm}(s) e^{-i g^{ \pm}\left(s, p^{\prime}\right)}
$$


where

$$
g^{ \pm}\left(s, p^{\prime}\right)=\int_{0}^{ \pm \infty} V_{1}\left(u+s, u p^{\prime}+\frac{1}{2} u^{2} E_{0}\right) d u .
$$

Thus, if we set $S_{D}(s)=\Omega_{D}^{+*}(s) \Omega_{D}^{-}(s)$, one has :

$$
S_{1}(s)=e^{-i g^{+}\left(s, p^{\prime}\right)} S_{D}(s) e^{i g^{-}\left(s, p^{\prime}\right)}
$$

\section{- Step 3 : High energy estimates}

Denote $\rho=\min (1, \delta)$. We have the following estimations, (the proof is exactly the same as in ([15], Lemma 3) for time-independent potentials).

\section{Lemma 6.}

For $\lambda>>1$, we have :

$$
\begin{array}{r}
\left\|\left(V_{1}(t, x)-V_{1}\left(t,(t-s) p^{\prime}+\frac{1}{2}(t-s)^{2} E_{0}\right)\right) U_{D}(t, s) e^{i g^{ \pm}\left(s, p^{\prime}\right)} f_{\lambda, \omega}\right\| \\
\leq C(1+|(t-s) \sqrt{\lambda}|)^{-\frac{1}{2}-\rho} .
\end{array}
$$

$$
\begin{aligned}
& \left\|\left(R(t, s) \Omega_{D}^{ \pm}(s)-U_{D}(t, s)\right) e^{i g^{ \pm}\left(s, p^{\prime}\right)} f_{\lambda, \omega}\right\|=O\left(\lambda^{-\frac{1}{2}}\right) \text {, uniformly } \\
& \text { for } t, s \in \mathbb{R} \text {. }
\end{aligned}
$$

\section{- Step 4}

We denote $F(s, \lambda, \omega)=<\left[S_{1}(s), p\right] f_{\lambda, \omega}, g_{\lambda, \omega}>$. Using (2.24), we have :

$$
\begin{aligned}
F(s, \lambda, \omega) & =<\left[e^{-i g^{+}\left(s, p^{\prime}\right)} S_{D}(s) e^{i g^{-}\left(s, p^{\prime}\right)}, p\right] f_{\lambda, \omega}, g_{\lambda, \omega}> \\
& =<\left[S_{D}(s), p\right] e^{i g^{-}\left(s, p^{\prime}\right)} f_{\lambda, \omega}, e^{i g^{+}\left(s, p^{\prime}\right)} g_{\lambda, \omega}> \\
& =<\left[S_{D}(s)-1, p-\sqrt{\lambda} \omega\right] e^{i g^{-}\left(s, p^{\prime}\right)} f_{\lambda, \omega}, e^{i g^{+}\left(s, p^{\prime}\right)} g_{\lambda, \omega}> \\
& =<\left(S_{D}(s)-1\right) e^{i g^{-}\left(s, p^{\prime}\right)}(p f)_{\lambda, \omega}, e^{i g^{+}\left(s, p^{\prime}\right)} g_{\lambda, \omega}> \\
& -<\left(S_{D}(s)-1\right) e^{i g^{-}\left(s, p^{\prime}\right)} f_{\lambda, \omega}, e^{i g^{+}\left(s, p^{\prime}\right)}(p g)_{\lambda, \omega}> \\
& :=F_{1}(s, \lambda, \omega)-F_{2}(s, \lambda, \omega) .
\end{aligned}
$$

First, let us study $F_{1}(s, \lambda, \omega)$. Writing $S_{D}(s)-1=\left(\Omega_{D}^{+}(s)-\Omega_{D}^{-}(s)\right)^{*} \Omega_{D}^{-}(s)$ and using

$$
\begin{array}{r}
\Omega_{D}^{+}(s)-\Omega_{D}^{-}(s)=i \int_{-\infty}^{+\infty} R(s, t)\left[V_{1}(t, x)-V_{1}\left(t,(t-s) p^{\prime}+\right.\right. \\
\left.\left.\frac{1}{2}(t-s)^{2} E_{0}\right)\right] U_{D}(t, s) d t
\end{array}
$$


we obtain :

$$
\begin{array}{r}
S_{D}(s)-1=-i \int_{-\infty}^{+\infty} U_{D}(t, s)^{*}\left[V_{1}(t, x)-V_{1}\left(t,(t-s) p^{\prime}+\frac{1}{2}(t-s)^{2} E_{0}\right)\right] \\
R(t, s) \Omega_{D}^{-}(s) d t
\end{array}
$$

Thus,

where :

$$
\begin{aligned}
F_{1}(s, \lambda, \omega)=-i & \int_{-\infty}^{+\infty}<R(t, s) \Omega_{D}^{-}(s) e^{i g^{-}\left(s, p^{\prime}\right)}(p f)_{\lambda, \omega},\left[V_{1}(t, x)-\right. \\
& \left.V_{1}\left(t,(t-s) p^{\prime}+\frac{1}{2}(t-s)^{2} E_{0}\right)\right] U_{D}(t, s) e^{i g^{+}\left(s, p^{\prime}\right)} g_{\lambda, \omega}>d t \\
=-i & \int_{-\infty}^{+\infty}<U_{D}(t, s) e^{i g^{-}\left(s, p^{\prime}\right)}(p f)_{\lambda, \omega},\left[V_{1}(t, x)-\right. \\
& \left.V_{1}\left(t,(t-s) p^{\prime}+\frac{1}{2}(t-s)^{2} E_{0}\right)\right] U_{D}(t, s) e^{i g^{+}\left(s, p^{\prime}\right)} g_{\lambda, \omega}>d t \\
& +R_{1}(s, \lambda, \omega),
\end{aligned}
$$

$$
\begin{gathered}
R_{1}(s, \lambda, \omega)=-i \int_{-\infty}^{+\infty}<\left[R(t, s) \Omega_{D}^{-}(s)-U_{D}(t, s)\right] e^{i g^{-}\left(s, p^{\prime}\right)}(p f)_{\lambda, \omega} \\
{\left[V_{1}(t, x)-V_{1}\left(t,(t-s) p^{\prime}+\frac{1}{2}(t-s)^{2} E_{0}\right)\right] U_{D}(t, s) e^{i g^{+}\left(s, p^{\prime}\right)} g_{\lambda, \omega}>d t}
\end{gathered}
$$

By Lemma 6, it is clear that $R_{1}(s, \lambda, \omega)=O\left(\lambda^{-1}\right)$. Thus, writing $t=\frac{\tau}{\sqrt{\lambda}}+s$, we obtain :

$$
\begin{aligned}
F_{1}(s, \lambda, \omega)=- & \frac{i}{\sqrt{\lambda}} \int_{-\infty}^{+\infty}<U_{D}\left(\frac{\tau}{\sqrt{\lambda}}+s, s\right) e^{i g^{-}\left(s, p^{\prime}\right)}(p f)_{\lambda, \omega}, \\
& \left(V_{1}\left(\frac{\tau}{\sqrt{\lambda}}+s, x\right)-V_{1}\left(\frac{\tau}{\sqrt{\lambda}}+s, \frac{\tau}{\sqrt{\lambda}} p^{\prime}+\frac{\tau^{2}}{2 \lambda} E_{0}\right)\right) \\
& U_{D}\left(\frac{\tau}{\sqrt{\lambda}}+s, s\right) e^{i g^{+}\left(s, p^{\prime}\right)} g_{\lambda, \omega}>d \tau+O\left(\lambda^{-1}\right) .
\end{aligned}
$$

Denote by $f_{1}(\tau, s, \lambda, \omega)$ the integrand of the (R.H.S) of (2.28). By Lemma 6 (i),

$$
\left|f_{1}(\tau, s, \lambda, \omega)\right| \leq C(1+|\tau|)^{-\frac{1}{2}-\rho} .
$$

So, by Lebesgue's theorem, to obtain the asymptotics of $F_{1}(s, \lambda, \omega)$, it suffices to determine $\lim _{\lambda \rightarrow+\infty} f_{1}(\tau, s, \lambda, \omega)$.

Let us denote :

$$
U^{ \pm}\left(t, s, p^{\prime}\right)=e^{i \int_{t}^{ \pm \infty}} V_{1}\left(u+s, u p^{\prime}+\frac{1}{2} u^{2} E_{0}\right) d u .
$$


We have :

$$
\begin{gathered}
f_{1}(\tau, s, \lambda, \omega)=<e^{-i \frac{\tau}{\sqrt{\lambda}} B_{0}} U^{-}\left(\frac{\tau}{\sqrt{\lambda}}, s, p^{\prime}\right)(p f)_{\lambda, \omega}, \\
\left(V_{1}\left(\frac{\tau}{\sqrt{\lambda}}+s, x\right)-V_{1}\left(\frac{\tau}{\sqrt{\lambda}}+s, \frac{\tau}{\sqrt{\lambda}} p^{\prime}+\frac{\tau^{2}}{2 \lambda} E_{0}\right)\right) \\
e^{-i \frac{\tau}{\sqrt{\lambda}} B_{0}} U^{+}\left(\frac{\tau}{\sqrt{\lambda}}, s, p^{\prime}\right) g_{\lambda, \omega}>.
\end{gathered}
$$

Using the Avron-Herbst formula (2.2), we deduce that :

$$
\begin{aligned}
& f_{1}(\tau, s, \lambda, \omega)=<e^{-i \frac{\tau}{2 \sqrt{\lambda}} p^{2}} U^{-}\left(\frac{\tau}{\sqrt{\lambda}}, s, p^{\prime}\right)(p f)_{\lambda, \omega}, \\
& \left(V_{1}\left(\frac{\tau}{\sqrt{\lambda}}+s, x+\frac{\tau^{2}}{2 \lambda} E_{0}\right)-V_{1}\left(\frac{\tau}{\sqrt{\lambda}}+s, \frac{\tau}{\sqrt{\lambda}} p^{\prime}+\frac{\tau^{2}}{2 \lambda} E_{0}\right)\right) \\
& e^{-i \frac{\tau}{2 \sqrt{\lambda}} p^{2}} U^{+}\left(\frac{\tau}{\sqrt{\lambda}}, s, p^{\prime}\right) g_{\lambda, \omega}>.
\end{aligned}
$$

Then, we obtain :

$$
\begin{gathered}
f_{1}(\tau, s, \lambda, \omega)=<e^{-i \frac{\tau}{2 \sqrt{\lambda}}(p+\sqrt{\lambda} \omega)^{2}} U^{-}\left(\frac{\tau}{\sqrt{\lambda}}, s, p^{\prime}+\sqrt{\lambda} \omega\right) p f \\
\left(V_{1}\left(\frac{\tau}{\sqrt{\lambda}}+s, x+\frac{\tau^{2}}{2 \lambda} E_{0}\right)-V_{1}\left(\frac{\tau}{\sqrt{\lambda}}+s, \frac{\tau}{\sqrt{\lambda}}\left(p^{\prime}+\sqrt{\lambda} \omega\right)+\frac{\tau^{2}}{2 \lambda} E_{0}\right)\right) \\
e^{-i \frac{\tau}{2 \sqrt{\lambda}}(p+\sqrt{\lambda} \omega)^{2}} U^{+}\left(\frac{\tau}{\sqrt{\lambda}}, s, p^{\prime}+\sqrt{\lambda} \omega\right) g>
\end{gathered}
$$

Since

$$
e^{-i \frac{\tau}{2 \sqrt{\lambda}}(p+\sqrt{\lambda} \omega)^{2}}=e^{-i \frac{\tau \sqrt{\lambda}}{2}} e^{-i \tau \omega \cdot p} e^{-i \frac{\tau}{2 \sqrt{\lambda}} p^{2}}
$$

we have

$$
\begin{gathered}
f_{1}(\tau, s, \lambda, \omega)=<e^{-i \frac{\tau}{2 \sqrt{\lambda}} p^{2}} U^{-}\left(\frac{\tau}{\sqrt{\lambda}}, s, p^{\prime}+\sqrt{\lambda} \omega\right) p f \\
\left(V_{1}\left(\frac{\tau}{\sqrt{\lambda}}+s, x+\tau \omega+\frac{\tau^{2}}{2 \lambda} E_{0}\right)-V_{1}\left(\frac{\tau}{\sqrt{\lambda}}+s, \frac{\tau}{\sqrt{\lambda}}\left(p^{\prime}+\sqrt{\lambda} \omega\right)+\frac{\tau^{2}}{2 \lambda} E_{0}\right)\right) \\
e^{-i \frac{\tau}{2 \sqrt{\lambda}} p^{2}} U^{+}\left(\frac{\tau}{\sqrt{\lambda}}, s, p^{\prime}+\sqrt{\lambda} \omega\right) g>
\end{gathered}
$$

Since $\left.\mid V_{1}\left(u+s, u\left(p^{\prime}+\sqrt{\lambda} \omega\right)+\frac{1}{2} u^{2} E_{0}\right)\right) \mid \leq C\left(u^{2}+1\right)^{-\delta} \in L^{1}\left(\mathbb{R}^{+}, d u\right)$, it is easy to show (using Lebesgue's theorem again) that :

$$
s-\lim _{\lambda \rightarrow+\infty} U^{ \pm}\left(\frac{\tau}{\sqrt{\lambda}}, s, p^{\prime}+\sqrt{\lambda} \omega\right)=1 .
$$

Then,

$$
\lim _{\lambda \rightarrow+\infty} f_{1}(\tau, s, \lambda, \omega)=<p f,\left(V_{1}(s, x+\tau \omega)-V_{1}(s, \tau \omega)\right) g>
$$


So, we have obtained :

$$
\begin{aligned}
F_{1}(s, \lambda, \omega)= & -\frac{i}{\sqrt{\lambda}}<p f,\left(\int_{-\infty}^{+\infty}\left(V_{1}(s, x+\tau \omega)-V_{1}(s, \tau \omega)\right) d \tau\right) g> \\
& +o\left(\frac{1}{\sqrt{\lambda}}\right) .
\end{aligned}
$$

In the same way, we obtain

$$
\begin{aligned}
F_{2}(s, \lambda, \omega)=- & \frac{i}{\sqrt{\lambda}}<f,\left(\int_{-\infty}^{+\infty}\left(V_{1}(s, x+\tau \omega)-V_{1}(s, \tau \omega)\right) d \tau\right) p g> \\
& +o\left(\frac{1}{\sqrt{\lambda}}\right)
\end{aligned}
$$

so

$$
\begin{aligned}
F(s, \lambda, \omega) & =F_{1}(s, \lambda, \omega)-F_{2}(s, \lambda, \omega) \\
& =\frac{1}{\sqrt{\lambda}}<f,\left(\int_{-\infty}^{+\infty} \partial_{x} V_{1}(s, x+\tau \omega) d \tau\right) g>+o\left(\frac{1}{\sqrt{\lambda}}\right) .
\end{aligned}
$$

Using (2.19) and $\partial_{x} V(s, x+\tau \omega)=e^{-i c(s) \cdot p} \partial_{x} V_{1}(s, x+\tau \omega) e^{i c(s) \cdot p}$, we obtain :

$$
F(s, \lambda, \omega)=\frac{1}{\sqrt{\lambda}}<\Phi,\left(\int_{-\infty}^{+\infty} \partial_{x} V(s, x+\tau \omega) d \tau\right) \Psi>+o\left(\frac{1}{\sqrt{\lambda}}\right)
$$

\section{References}

[1] S. Arians, Geometric approach to inverse scattering for the Schrödinger equation with magnetic and electric potentials, J. Math. Phys. 38 (1997), no. 6, 2761-2773.

[2] J. E. Avron and I. W. Herbst, Spectral and scattering theory for Schrödinger operators related to the Stark effect, Comm. Math. Phys. 52 (1977) 239-254.

[3] H. L. Cycon, R. G. Froese, W. Kirsch, and B. Simon, Schrödinger operators with application to quantum mechanics and global geometry, Texts and Monographs in Physics, Springer Study Edition. Springer Verlag, Berlin, 1987.

[4] V. Enss and R. Weder, The geometrical approach to multidimensional inverse scattering, J. Math. Phys. 36 (1995), no. 8, 3902-3921.

[5] A. Galtbayar, A. Jensen, and K. Yajima, Local time decay of solutions to Schrödinger equations with time-periodic potentials, J. Statist. Phys. 116 (2004), no. 1-4, 231-282.

[6] S. Helgason, The Radon Transform, Progress in Mathematics 5, Birkhäuser, 1980.

[7] J. S. Howland, Two problems with time-dependent Hamiltonians, Mathematical Methods and Application of Scattering Theory, ed. J. A. DeSanto, A. W. Saenz and W.W. Zachary, Lecture Notes in Physics, 130, Springer, 1980.

[8] H. T. Ito, An inverse scattering problem for Dirac equations with time-dependent electromagnetic potential, Publ. Res. Inst. Math. Sci. 34 (1998), no. 4, 355-381.

[9] W. Jung, Geometric approach to inverse scattering for Dirac equation, J. Math. Phys. 36 (1995), no. 8, 3902-3921.

[10] H. Kitada and K. Yajima, A scattering theory for time-dependent long-range potentials, Duke Math. J. 49 (1982), no. 2, 341-376.

[11] _ Remarks on our paper : A scattering theory for time-dependent long-range potentials, Duke Math. J. 50, (1983), no. 4, 1005-1016. 
[12] J. S. Moller, Two-body short-range systems in a time-periodic electric field, Duke Math. J. 105 (2000), no. 1, 135-166.

[13] F. Nicoleau, A stationary approach to inverse scattering for Schrödinger operators with first order perturbation, Comm. Partial Differential Equations 22 (1997), no. 3-4, 527-553.

[14] , An inverse scattering problem with the Aharonov-Bohm effect, J. Math. Phys. 41 (2000), no. 8, 5223-5237.

[15] _ Inverse scattering for Stark Hamiltonians with short-range potentials, Asymptot. Anal. 35 (2003), no. 3-4, 349-359.

[16] M. Reed and R. Simon, Methods of mathematical physics, Vol. 2, Academic Press, 1978.

[17] R. Weder, Multidimensional inverse scattering in an electric field, J. Funct. Anal. 139 (1996), no. 2, 441-465.

[18] _ Inverse scattering for $N$-body systems with time dependent potentials, Inverse Problems of Wave Propagation and Diffraction, Eds. G. Chavent, P. C. Sabatier, Lecture Notes in Physics 486, Springer Verlag, 1997.

[19] , The Aharonov-Bohm effect and time-dependent inverse scattering theory, Inverse Problems 18 (2002), no. 4, 1041-1056.

[20] _ Inverse scattering at a fixed quasi-energy for potentials periodic in time, Inverse Problems 20 (2004), no. 3, 893-917.

[21] K. Yajima, Time periodic Schrödinger equations, Topics in the theory of Schrödinger equations, World Scientific Publishing Co. Pte. Ltd, 2004.

[22] K. Yokoyama, Mourre theory for time-periodic systems, Nagoya Math. J. 149 (1998) 193-210.

Université de Nantes, Département de Mathématiques, Laboratoire Jean Leray, UMR CNRS-UN 6629, 2, RUE DE LA Houssinière BP 92208 , F-44322 NANTES CEdex 03, FRANCE

E-mail address: nicoleau@math.univ-nantes.fr 